\title{
Some Thermodynamic Aspects of Black Holes and Singularities
}

\author{
C. O. LOUSTO* \\ Department of Physics, University of Utah, \\ 201 JFB, Salt Lake City, UT 84112, USA
}

(September 26, 2018)

\begin{abstract}
We review and correct the classical critical exponents characterizing the transition from negative to positive black hole's heat capacity at high chargeangular momentum. We discuss the stability properties of black holes as a thermodynamic system in equilibrium with a radiation bath (canonical ensamble) by using the Helmholtz free energy potential. We finally analytically extend the analysis to negative mass holes and study its thermodynamical stability behavior.
\end{abstract}

04.70.Dy,05.70.Jk

*Electronic Address: lousto@mail.physics.utah.edu 


\section{INTRODUCTION}

The Kerr-Newman geometry (written in Boyer-Lindquist coordinates)

$$
\begin{aligned}
d s^{2}=- & \left(\Delta-a^{2} \sin ^{2} \theta\right) q^{-2} d t^{2}-4 M r a \sin ^{2} \theta q^{-2} d t d \varphi+\frac{q^{2}}{\Delta} d r^{2} \\
& +q^{2} d \theta^{2}+\left[\left(r^{2}+a^{2}\right)^{2}-\Delta a^{2} \sin ^{2} \theta\right] q^{-2} \sin ^{2} \theta d \varphi^{2}
\end{aligned}
$$

where

$$
\Delta=r^{2}-2 M r+a^{2}+Q^{2} \text { and } q^{2}=r^{2}+a^{2} \cos ^{2} \theta
$$

represents the general stationary black Hole solution of Einstein equations with an electromagnetic source. It is fully characterized by three parameters: Total energy $M$, Charge $Q$, and Angular Momentum $J$.

The more important geometrical property of the black hole for us will be its area $A_{+}$, defined as the area of the event horizon, located at a radial coordinate $r_{+}$

$$
A_{+}=4 \pi\left(r_{+}^{2}+a^{2}\right), \quad a=\frac{J}{M}
$$

and where, more explicitly, $r_{ \pm}=M \pm \sqrt{M^{2}-a^{2}-Q^{2}}\left(r_{-}\right.$being the internal or Cauchy horizon).

The connection with thermodynamics can be made by the identification of this geometrical quantity with the black hole entropy Ref. [1] (we hereafter take units in which $G=1$, $c=1$ and the Boltzmann constant, $k_{B}=1$ )

$$
S_{B H}=\frac{1}{4} A_{+} .
$$

The independent derivation of the black hole radiation temperature due to quantum effects in Ref. [2], gave a firm foundation to the thermodynamic interpretation of the otherwise mechanical laws for black holes [It also allowed to determine the exact proportionality factor in the above equation.]

Inverting $\mathrm{Eq}(3)$ we obtain the fundamental relation

$$
M=\left(\frac{S}{4 \pi}+\frac{\pi J^{2}}{S}+\frac{Q^{2}}{2}+\frac{\pi Q^{4}}{4 S}\right)^{1 / 2}
$$

which upon differentiation allow us to obtain the first law

$$
d M=T d S+\vec{\Omega} \cdot d \vec{J}+\Phi d Q .
$$

Hence we identify the temperature, angular velocity and electric potential

$$
T=\left.\frac{\partial M}{\partial S}\right|_{J Q}=\frac{1}{4 \pi} \frac{r_{+}-r_{-}}{\left(r_{+}^{2}+a^{2}\right)}, \quad \Omega=\left.\frac{\partial M}{\partial J}\right|_{S Q}=\frac{a}{r_{+}^{2}+a^{2}}, \quad \Phi=\left.\frac{\partial M}{\partial Q}\right|_{S J}=\frac{Q r_{+}}{r_{+}^{2}+a^{2}} .
$$

Davies [3] was the first to observe that if we suppose that a rotating charged black hole is held in equilibrium at some temperature $T$, with a surrounding heat bath; the full thermal capacity keeping $J$ and $Q$ constant, 


$$
C_{J Q}=\left.T \frac{\partial S}{\partial T}\right|_{J Q}=\frac{M T S^{3}}{\pi J^{2}+\frac{\pi}{4} Q^{4}-T^{2} S^{3}}
$$

goes from negative values for a Schwarzschild black hole, $C_{S c h}=-M / T$, to positive values for a nearly extreme Kerr-Newman black hole, $C_{E K N} \sim \sqrt{M^{4}-J^{2}-M^{2} Q^{2}} \rightarrow 0^{+}$through an infinite discontinuity.

In Ref. [1] we tried to understand this divergence of the heat capacity as indicative of critical phenomena in the thermodynamic description of black holes. We recall here that near the critical point (or curve in our case) one can define critical exponents that characterize the behavior of the relevant thermodynamic functions near criticality:

For the specific heat at constant $J$ and $Q$

$$
C_{J Q}=\left.T \frac{\partial S}{\partial T}\right|_{J Q} \sim\left\{\begin{array}{ll}
\left(T_{c}-T\right)^{-\alpha^{\prime}}, & \text { for } J=J_{c} \text { and } Q=Q_{c} \\
\left(J-J_{c}\right)^{-\varphi} \text { or }\left(Q-Q_{c}\right)^{-\varphi} & \text { for } T=T_{c}
\end{array} .\right.
$$

For the equation of state

$$
\Omega-\Omega_{c} \text { or } \Phi-\Phi_{c} \sim\left\{\begin{array}{ll}
\left(T_{c}-T\right)^{\beta^{\prime}}, & \text { for } J=J_{c} \text { and } Q=Q_{c} \\
\left(J-J_{c}\right)^{1 / \delta} \text { or }\left(Q-Q_{c}\right)^{1 / \delta} & \text { for } T=T_{c}
\end{array}\right. \text {. }
$$

For the isothermal capacitance or moment of inertia

$$
K_{T Q, T J}^{-1}=\left.\frac{\partial \Omega}{\partial J}\right|_{T Q} \text { or }\left.\frac{\partial \Phi}{\partial Q}\right|_{T J} \sim\left\{\begin{array}{l}
\left(T_{c}-T\right)^{-\gamma^{\prime}}, \quad \text { for } J=J_{c} \text { and } \begin{array}{l}
Q=Q_{c} \\
\left(J-J_{c}\right)^{1 / \delta-1} \text { or }\left(Q-Q_{c}\right)^{1 / \delta-1} \text { for }
\end{array} \quad T=T_{c}
\end{array} .\right.
$$

And for the entropy

$$
S-S_{c} \sim\left\{\begin{array}{ll}
\left(T_{c}-T\right)^{1-\alpha^{\prime}}, & \text { for } J=J_{c} \text { and } Q=Q_{c} \\
\left(J-J_{c}\right)^{\psi} \text { or }\left(Q-Q_{c}\right)^{\psi} & \text { for } T=T_{c}
\end{array} .\right.
$$

Primed exponents refer to temperatures below $T_{c}$, that is where heat capacities are positive and black holes can be kept in equilibrium with the radiation bath. Other two heat capacities, $C_{\Omega Q}$ and $C_{J \Phi}$, also exhibit a singular behavior along two other critical curves as shown in Ref. [5, 4].

The critical exponents defined above are not all independent but are related by the scaling laws

$$
\begin{gathered}
\alpha^{\prime}+2 \beta^{\prime}+\gamma^{\prime}=2, \quad \alpha^{\prime}+\beta^{\prime}(\delta+1)=2, \\
\gamma^{\prime}(\delta+1)=\left(2-\alpha^{\prime}\right)(\delta-1), \quad \gamma=\beta^{\prime}(\delta-1), \\
\left(2-\alpha^{\prime}\right)(\delta \psi-1)+1=\left(1-\alpha^{\prime}\right) \delta, \quad \varphi+2 \psi-\delta^{-1}=1 .
\end{gathered}
$$

These relations can be derived supposing the thermodynamic potentials are homogeneous functions of their variables near criticality. Since this law has macroscopic important consequences and cannot be derived from the other four, we call it the fourth law of thermodynamics.

The paper is organized as follows. In Sec. II we rederive the classical critical exponents for black holes and rediscuss its equilibrium properties in terms of the Helmholtz potential. In Sec. III we extend the analysis to formally include negative mass objects. We end the paper with a discussion of these results. 


\section{CRITICAL EXPONENTS AND STABILITY ANALYSIS}

From the first of Eqs. (7) and Eq. (5) we can obtain $T=T(S, J, Q)$, [For the sake of simplicity we will collect the variables into $x=(J, Q)$.] We then make the following Taylor expansion near the critical curve

$$
T(S, x)=T_{c}+\left.\frac{\partial T}{\partial S}\right|_{c, x}\left(S-S_{c}\right)+\left.\frac{\partial T}{\partial x}\right|_{c, S}\left(x-x_{c}\right)+\left.\frac{1}{2} \frac{\partial^{2} T}{\partial S^{2}}\right|_{c, x}\left(S-S_{c}\right)^{2}+\ldots
$$

We first note at the black hole critical curve we have $\partial T /\left.\partial S\right|_{c, x}=0$, since that is $T /\left.C_{J Q}\right|_{c}$ what vanishes at the critical point (see Ref. [⿴囗十 for the explicit expression of $T_{c}$ )

Near the critical curve and for $x=x_{c}$, we then get

$$
S-S_{c} \approx \pm \sqrt{\frac{2}{\left.\frac{-\partial^{2} T}{\partial S^{2}}\right|_{c, x}}} \sqrt{T_{c}-T} \quad \Rightarrow \quad \alpha^{\prime}=\frac{1}{2}
$$

where the last implication is obtained upon comparison with Eq. (12).

For the heat capacity we find

$$
\left.C_{x} \approx T_{c} \frac{\Delta S}{\Delta T}\right|_{x} \approx \operatorname{Sign}\left[S_{c}-S\right] \sqrt{\frac{2}{\left.\frac{-\partial^{2} T}{\partial S^{2}}\right|_{c, x}}} \frac{T_{c}}{\sqrt{T_{c}-T}} \quad \Rightarrow \quad \alpha^{\prime}=\frac{1}{2},
$$

this time the comparison is made with Eq. (9).

Now letting be $T=T_{c}$ we get

$$
S-S_{c} \approx \pm \sqrt{\frac{-\left.2 \frac{\partial T}{\partial x}\right|_{c, S}}{\left.\frac{-\partial^{2} T}{\partial S^{2}}\right|_{c, x}}} \sqrt{x_{c}-x} \quad \Rightarrow \quad \psi=\frac{1}{2}
$$

where now we looked at the definition of $\psi$ given in Eq. (12).

And finally, for the heat capacity we obtain

$$
\left.C_{x} \approx T_{c} \frac{\Delta S}{\Delta T}\right|_{x} \approx \operatorname{Sign}\left[S_{c}-S\right] T_{c} \sqrt{\frac{2}{\left.\frac{\partial^{2} T}{\partial S^{2}}\right|_{c, x} \times\left.\frac{\partial T}{\partial x}\right|_{c, S}}} \frac{1}{\sqrt{x_{c}-x}} \quad \Rightarrow \quad \varphi=\frac{1}{2} .
$$

where we used the second row of Eq. (9).

To find the other exponents we instead consider the Taylor development of the equation of state. We shall use now the notation $y=(\Omega, \Phi)$ for the conjugate variables of $x=(J, Q)$. Then we write

$$
x(T, y)=x_{c}+\left.\frac{\partial x}{\partial y}\right|_{c, T}\left(y-y_{c}\right)+\left.\left.\frac{\partial x}{\partial T}\right|_{c, y}\left(T-T_{c}\right) \frac{1}{2} \frac{\partial^{2} x}{\partial y^{2}}\right|_{c, T}\left(y-y_{c}\right)^{2}+\ldots
$$

Note at the black hole critical curve we have $\partial x /\left.\partial y\right|_{c, T}=0$, since the inverse of the isothermal capacitance or moment of inertia vanishes at the critical point [5].

Again, near criticality, for $x=x_{c}$, we then get 


$$
y-y_{c} \approx \pm \sqrt{\frac{-\left.2 \frac{\partial x}{\partial T}\right|_{c, y}}{\left.\frac{-\partial^{2} x}{\partial y^{2}}\right|_{c, T}}} \sqrt{T_{c}-T} \quad \Rightarrow \quad \beta^{\prime}=\frac{1}{2}
$$

where the last implication is found from Eq. (10).

For the inverse of the moment of inertia or capacitance we find

$$
\left.K_{T}^{-1} \approx \frac{\Delta y}{\Delta x}\right|_{T} \approx \operatorname{Sign}\left[y_{c}-y\right] \sqrt{\frac{2}{\left.\frac{-\partial^{2} x}{\partial y^{2}}\right|_{c, T} \times\left.\frac{\partial x}{\partial T}\right|_{c, y}}} \frac{1}{\sqrt{T_{c}-T}} \Rightarrow \gamma^{\prime}=\frac{1}{2}
$$

the comparison was made with Eq. (11).

Now, taking $T=T_{c}$, we get

$$
y-y_{c} \approx \pm \sqrt{\frac{2}{\left.\frac{-\partial^{2} x}{\partial y^{2}}\right|_{c, T}}} \sqrt{x_{c}-x} \quad \Rightarrow \quad \delta=2,
$$

where we looked at Eq. (10).

And finally, we obtain

$$
\left.K_{T}^{-1} \approx \frac{\Delta y}{\Delta x}\right|_{T} \approx \operatorname{Sign}\left[y_{c}-y\right] \sqrt{\frac{2}{\left.\frac{-\partial^{2} x}{\partial y^{2}}\right|_{c, T}}} \frac{1}{\sqrt{x_{c}-x}} \Rightarrow \delta=2 .
$$

where we made use of the second row of Eq. (11).

The above considerations are general and apply to the charged and rotating black holes (i.e. Kerr-Newman black holes represented by metric (1)). Although we have computed the exponents for the canonical ensemble at $J, Q$ fixed the analysis also apply to the other two transitions we considered in Ref. [四], at $\Omega, Q$ or $J, \Phi$ fixed by properly identifying the variables $x$ and $y$ above and the thermodynamical potentials as in [4].

We can check the correctness of the above derived exponents by considering nonrotating charged black holes (i.e. Reissner-Nordström ones) where equations simplify enough to compute explicitly the behavior near the critical point. In particular, it is easy to check form Eqs. (7) that the equation of state can be written as

$$
4 \pi T Q=\Phi-\Phi^{3}
$$

The critical values of the variables for the Reissner-Nordström black hole are given by

$$
\Phi_{c}=\frac{1}{\sqrt{3}}, \quad Q_{c} T_{c}=\frac{1}{6 \pi \sqrt{3}} .
$$

This allows us to write the equation of state as

$$
\left(\Phi-\Phi_{c}\right)^{2}\left(\Phi+2 \Phi_{c}\right)=\frac{1}{6 \pi \sqrt{3}}\left(1-\frac{T Q}{T_{c} Q_{c}}\right)
$$

what near the critical point behaves like

$$
\Phi-\Phi_{c} \approx \pm \frac{1}{\sqrt{18 \pi}} \begin{cases}\left(T_{c}-T\right)^{1 / 2}, & \text { for } Q=Q_{c} \\ \left(Q_{c}-Q\right)^{1 / 2}, & \text { for } T=T_{c}\end{cases}
$$


We thus see that $\beta^{\prime}=1 / 2$ and $\delta=2$.

Using the above result for the behavior of $\Phi$ near criticality, and given

$$
S=\pi \frac{Q^{2}}{\Phi^{2}}, \quad K_{T Q}^{-1}=-\frac{4 \pi T}{3 \Phi^{2}-1}, \quad C_{Q}=\frac{4 \pi^{2} Q^{3}}{\Phi^{3}\left(3 \Phi^{2}-1\right)},
$$

it is easy to check that all the exponents we get are those given by Eqs. (15)-(23).

Let us now analyze the stability properties of black holes in equilibrium with the thermal bath. The appropriate potential to study the canonical ensamble is the Helmholtz free energy

$$
F(T, \vec{J}, Q)=M-T S=T S+2 \vec{\Omega} \cdot \vec{J}+\Phi Q
$$

where in the last equality we have used the mass formula of Ref. [6].

From this potential can be derived all the thermodynamical variables of our interest

$$
\Phi=\left.\frac{\partial F}{\partial Q}\right|_{T J}, \quad \Omega=\left.\frac{\partial F}{\partial J}\right|_{T Q}, \quad S=-\left.\frac{\partial F}{\partial T}\right|_{J Q} .
$$

and

$$
C_{J Q}=-\left.T \frac{\partial^{2} F}{\partial T^{2}}\right|_{J Q}, \quad K_{T Q}^{-1}=\left.\frac{\partial^{2} F}{\partial J^{2}}\right|_{T Q}, \quad K_{T J}^{-1}=\left.J \frac{\partial^{2} F}{\partial Q^{2}}\right|_{T J} .
$$

From Eqs. (15)-(23) we see that near criticality, first derivatives of the potential are continuous (hence also the mass, what implies zero latent heat), while second derivatives diverge.

Now, by use of the equation of state we can, in principle, rewrite part of the Helmholtz potential as a function $f$ of the variables $T, \vec{\Omega}$, and $\Phi$, in such a way that

$$
F=f(T, \vec{\Omega}, \Phi)+\vec{\Omega} \cdot \vec{J}+\Phi Q
$$

We thus arrived to a form that looks like the effective potential of an antiferromagnet (due to the plus sign of the last two addends) in an external field $x=(Q, \vec{J})$ and having an order parameter $y=(\Phi, \vec{\Omega})$ with two (or in general four) components, which is the conjugate variable to $x$, i.e.

$$
y=\left.\frac{\partial F}{\partial x}\right|_{T},
$$

corresponding to the first two equations of (30).

The equation of state is given by the extremum condition of the potential

$$
\left.\frac{\partial F}{\partial y}\right|_{T}=0=\left.\frac{\partial f}{\partial y}\right|_{T}+x
$$

Again, to be more explicit we can deal with the simpler case of nonrotating charged black holes. In this case by use of Eqs. (28) and (24) we obtain

$$
F(T, \Phi, Q)=\frac{\left(1-\Phi^{2}\right)^{2}}{16 \pi T}+Q \Phi
$$


We have plotted the equation of state (24) in figures (1) and (2). In the first figure we fist observe that the charge presents a maximum at the same value $\Phi_{c}=1 / \sqrt{3}$, its value being independent of the temperature. If we consider a fixed value of the charge, like the one shown in the figure, we see that at low temperatures there are two possible values of $\Phi$, denoted by $I$ and $I I$. As we rise the temperature of the system, those two states merge together at the critical temperature $T_{c}=1 /(6 \pi \sqrt{3} Q)$. Above this temperature, no equilibrium state is possible. One can also see that a state in the branch $I$ of the equation of state has a higher mass than the corresponding state labeled by $I I$ since $\Phi_{I I}>\Phi_{I}$ and from Eqs. (7)and (24) we have

$$
\Delta M=M_{I I}-M_{I}=\frac{\Phi_{I}^{4}-\Phi_{I I}^{4}}{T} \leq 0 .
$$

For completeness, the electrostatic potential can be found by applying Cardano's formulae to the equation of state (24)

$$
\Phi_{I}=\frac{2}{\sqrt{3}} \cos \left(\frac{\theta}{3}\right), \quad \Phi_{I I}=\frac{2}{\sqrt{3}} \cos \left(\frac{\theta+4 \pi}{3}\right),
$$

where $\cos (\theta)=-(6 \pi \sqrt{3} T Q)$. $\Delta M$ ranges form $-1 /(8 \pi T)$ for $Q=0$ to zero for $Q=Q_{c}$.

Finally, when the charge of the black hole is negative we have the same plot reflected thought the origin to the third quadrant.

In the second figure we see that at a fixed value of the charge we can have any temperature from zero to $T_{c}$ by letting $\Phi$ vary in the range $(0,1)$ [The same happens for $T$ fixed; we have $0<Q<Q_{c}$.] i.e. $Q T \leq 1 /(6 \pi \sqrt{3})=Q_{c} T_{c}$. When negative charges are considered we have reflection of this figure to the fourth quadrant.

Further insight can be gained on the stability of the branches $I$ and $I I$ when we consider the Helmholtz potential (35) shown in figure (3). In fact, it comes out clearly from the figure that the branch $I I$ represents a minimum of the potential, while the branch $I$ is a maximum. Since black holes in $I$ have a higher mass than those in $I I$ they have a lower ratio $Q / M$ and they are in the branch closer to the uncharged black holes (i.e. Schwarzschild ones). In fact, this black holes possess a negative heat capacity that indicate they cannot be held in equilibrium with an infinite radiation bath. Depending on the sign of the perturbation they can evolve towards the stable minimum by losing mass (for instance, if they keep a temperature higher than that of the bath) or towards the Schwarzschild-like holes by indefinitely gaining mass in favor of the radiation bath (for instance, if they keep a lower temperature than the bath). On the other hand, black holes in branch $I I$ have a higher ratio $Q / M$ that makes their heat capacity positive, allowing them to be in equilibrium with radiation. As we rise the temperature, the two branches merge together and the stable state II disappears for $T>T_{c}$, making the hole evolve towards an infinite mass state.

We can thus compare this phenomenon with what happens in a magnet near criticality: At high temperature the magnet do not present any net magnetization $(\vec{M})$, but as we lower its temperature below it Curie point $\left(T=T_{c}\right)$ one observes a spontaneous magnetization to appear even at zero external applied field. Further lowering its temperature, makes to increase the magnetization. Two values of the magnetization $\pm \vec{M}$ are possible depending on the initial perturbation. The analogy with our black hole system here is limited since one of the two branches of the electrostatic field (our order parameter), the $\Phi_{I}$, is unstable. 
In this restricted sense, the transition between branch $I$ and $I I$ ends at $\Phi_{c}$ which can be called a critical point. $T_{c}$ now being the parameter that gives the change in the stability properties of highly charged-rotating black holes $\left(\Phi_{I I}\right)$.

\section{THERMODYNAMICS OF A HOLE WITH NEGATIVE MASS?}

In the above analysis we have supposed that $\Phi$ could not be bigger than one since this represents the extremal black hole. If we also consider negative charges this statement generalizes to $|\Phi| \leq 1$. But let us be curious enough to see what represents $|\Phi|>1$. To do so, we take the last equation in (四), and plug the explicit form of $r_{+}$into

$$
\Phi=\frac{Q}{M+\sqrt{M^{2}-Q^{2}}}
$$

to keep $\Phi$ real we consider $|M| \geq|Q|$. One can thus probe the following implications:

1) $|\Phi| \leq 1 \Rightarrow M \geq 0 \Rightarrow \operatorname{sign}\{\Phi\}=\operatorname{sign}\{Q\}$ and

2) $|\Phi| \geq 1 \Rightarrow M \leq 0 \Rightarrow \operatorname{sign}\{\Phi\}=-\operatorname{sign}\{Q\}$

[The analogous implications hold for the rotating (Kerr) hole and can be probed by use of the second equation in (7).]

We are thus dealing with negative mass objects. Let us also note that the Kerr-Newman metric (11) is invariant under the change of sign of its parameters and the radial coordinate

$$
M_{*} \rightarrow-M, \quad r_{*} \rightarrow-r, \quad Q_{*} \rightarrow-Q, \quad J_{*} \rightarrow-J
$$

Note that while the last two reflections have a natural interpretation, we usually do not consider negative mass objects due to its odd stability properties and the fact that the represent naked singularities [also, changing the sign of $r$ is not a covariant transformation, but for our following discussion of the thermodynamical variables this will not be relevant.]

If we extend the usual thermodynamic relations by the discrete $*$ symmetry we find that (considering now the quantity $M>0$ )

$$
r_{ \pm}\left(M_{*}\right)=r_{ \pm}(-M)=-r_{\mp}(M) .
$$

by direct use of the definitions. That is all the dependence on the mass we need to compute the thermodynamic variables given by Eqs. (28) and (7).

For the "intensive" variables

$$
T_{*}=-T\left(r_{-}\right)>T_{+}>0 ; \quad \Omega_{*}=\Omega\left(r_{-}\right)>\Omega_{+}>0 ; \quad \Phi_{*}=\Phi\left(r_{-}\right)>\Phi_{+}>0,
$$

and for the "extensive" variables

$$
0<S_{*}=S\left(r_{-}\right)<S_{+} ; \quad J_{*}=-J<0 ; \quad Q_{*}=-Q<0 .
$$

[where the inequalities for \pm quantities are due to $r_{+}(M)>r_{-}(M)$, and they become equalities in the extremal case.]

We are interested in the case of holes in equilibrium with a radiation bath, i.e. in the canonical ensamble. For that, we have to fix the temperature $T$, the charge $Q$ and the 
angular momentum $J$. We have thus to impose only the first two of the $*$ transformations (Eqs. (39)) to be able to deal with positive and negative mass states on the same foot at fixed $Q$ and $J$. As a result, negative mass thermodynamic states will have the last two quantities in Eqs. (41) and (42) with the sign opposed to that shown. Thus, keeping the positiveness of the angular momentum and charge, but negative angular velocity and electrostatic potential.

In the following we will discuss the simpler nonrotating charged case. Figure (đ) shows how the equation of state extended to all the four quadrants looks like. The region $0<\Phi<1$ in the first quadrant is what we have discussed in the last section. Its extention to negative charges is in the third quadrant. The negative mass states are characterized by $|\Phi|>1$ and are to the right or to the left of the abscissa axis according to its positive or negative charge respectively. We shall characterize this states by the label $I I I$. We first observe that at a given temperature according to the value we fix for the charge of the system we can have one two or three possible states. At absolute charges below the critical one we have three states. In addition to the $I$ and $I I$ of the last section we will also have a negative mass state. For $|Q|>\left|Q_{c}\right|$ only the state $I I I$ is possible (while there was not state in the last section analysis). And finally, for the critical value of the charge only two states are allowed. The equation of state crosses also three times the $\Phi$ axis. The points $\Phi= \pm 1$ correspond to the $M \rightarrow 0$ limit but in such a way that the ratio $Q / M \rightarrow \pm 1$, thus $Q \rightarrow 0$ too. The point in the origin corresponds to the infinite mass limit. Note that the full figure looks like the corresponding one for the mean field approximation to the Ising model with a non-zero applied external magnetic field and an infinite critical temperature [7].

In figure (5) we plot the Helmholtz potential that will help us to discuss the most relevant question of the stability of the negative mass objects. For a fixed positive charge $Q$ we have three qualitatively different kind of potential depending on the value of the temperature. For temperatures lower than the critical one, the potential has a minimum in $\Phi_{I I I} \leq-1$ corresponding to the negative mass sector, then a relative maximum at $0 \leq \Phi_{I}<1 / \sqrt{3}$ and a relative minimum at $1 / \sqrt{3}<\Phi_{I I} \leq 1$. We thus see that the sector $I I$ of the black holes are now a metastable state that could evolve (through a first order transition) to a negative mass state. As we rise the temperature to the critical one this transition will be smooth and of second order. Above the critical temperature, the metastable state disappears and only the negative mass object remains, which happens to be the most stable object of the ensamble. Since this object has a positive temperature, it could eventually emit more radiation than received form the bath, thus decreasing its mass, but as the absolute value of $M$ increases, this makes the emission temperature to decrease, below the bath temperature, thus stabilizing the object. Conversely, a fluctuation in the temperature of the object that make it decrease, would make it gain positive mass on the bath making its absolute value decrease and thus finally radiating at a temperature higher than that of the bath, reaching in that way the stable point again.

\section{DISCUSSION}

In the first part of the paper we have recomputed and corrected the critical exponents for highly rotating-charged black holes given in [8]. In Ref. [8] we have used them to guess the effective dimension of the system. This cannot be done here since we have computed only 
the thermodynamic exponents (Eqs. (15)-(23)), which are independent of the dimensionality of the system. Critical exponents related to the two point correlation function do depend on the dimensionality and can be used to determine it.

It is worth noting that critical phenomena have also been observed in numerical analysis of the collapse of a scalar field to form a black hole [9], and that the classical value $1 / 2$ for the exponents have also been found there [10].

The exponents we computed are classical. To improve them one should have a quantum version of the analysis, use the Renormalization Group and get the corrections to (15)(23). This can be done by considering the effective Hamiltonian derived from the Helmholtz potential and is under current research. The quantum analysis could eventually give us a physical interpretation of the negative energy states. In conclusion, although we treat semiclassical (neither astrophysical nor Planck scale) black holes, it seems that some new (perhaps quantum) phenomena appears below the critical temperature $T_{c}$.

In Refs. [11] it was introduced the concept of "spin entropy" and "spin temperature" for Kerr black holes in terms of the quantities defined on the internal horizon and it was studied its thermodynamics. In particular, $T_{-}<0$, was interpreted as reflecting the inversion of population like in usual spin systems. In our paper, on the other hand, thermodynamic quantities evaluated on the internal horizon appear as a consequence of extending the usual formulae for black holes to objects with negative mass described by the Kerr-Newman geometry. Its temperature is $-T_{-}$, thus defined positive, and they can be in equilibrium with an infinite radiation bath. There still remain, of course, to know if there is any object in nature that could (at least effectively) be described in such way.

\section{ACKNOWLEDGMENTS}

C.O.L was supported by the NSF grant PHY-95-07719 and by research founds of the University of Utah. 


\section{REFERENCES}

[1] J. D. Bekenstein, Phys. Rev., D7, 949 (1973); ibid, D7, 2333 (1973); ibid, D9, 3292 (1974).

[2] S. W. Hawking, Nature., 248, 30 (1974); Commun. Math. Phys., 43, 199 (1975).

[3] P. C. W. Davies, Proc. R. Soc. Lond., A353, 499 (1977).

[4] C. O. Lousto, Nucl. Phys., B410, 155 (1993). Erratum, ibid, B449, 433 (1995).

[5] D. Tranah and P. T. Landsberg, Collective Phenomena, 3, 81 (1980).

[6] L. Smarr, Phys. Rev. Lett., 30, 71 (1973).

[7] G. Parisi, Statistical Field Theory (Addison-Wesley, Redwood City, CA, 1988), Ch 3.

[8] C.O.Lousto, Phys.Rev., D51, (1995) 1733.

[9] M. Choptuik, Phys Rev. Lett, 70, 9 (1993).

[10] J. Pullin, Phys. Lett, A204, 7 (1995).

[11] A. Curir, Nouvo Cim., B51, 262 (1979); Europhys. Lett., 9, 609 (1989). 


\section{FIGURES}

FIG. 1. Isotherms for the Reissner-Nordström black hole. The dashed line gives the location of the maximum always occurring at $\Phi=1 / \sqrt{3}$. For a given constant value of the charge $Q>0$ we can have two, one or none values of $\Phi$. The low $\Phi$ branch corresponds to black holes with $C_{Q}<0$; while the high $\Phi$ corresponds to those with $C_{Q}>0$. The critical temperature is given by $T_{c}=(6 \pi \sqrt{3} Q)^{-1}$. For $Q<0$ we have the same figure reflected trough the origin to the third quadrant.

FIG. 2. The equation of state of a nonrotating charged black hole for different slices of the electrostatic potential. For a fixed value of the charge, the temperature is constrained to be less than the critical one. The same is true for the charge if we fix the temperature.

FIG. 3. The Helmholtz free energy for a fixed charge $Q>0$. At temperatures lower that the critical one there is a maximum at $\Phi_{I}$ and a minimum at $\Phi_{I I}$. At $T_{c}$ both coincide $\left(\Phi_{c}=1 / \sqrt{3}\right.$ there), and below there is neither a maximum nor a minimum in the considered range of $\Phi$.

FIG. 4. This is the extended equation of state of charged holes for all values of the electrostatic potential $\Phi$. Here branch $I$ is unstable, branch $I I$ represent metastable states and branch $I I I$ (corresponding to negative mass holes) are stable states. The Maxwell construction would avoid unstable and metastable sectors by simply joining branches $I I I$ along the $\Phi$ axis between the points $\Phi= \pm 1$. Otherwise a transition is possible between branches $I I$ and $I I I$ up to the critical value $\Phi_{c}= \pm 1 / \sqrt{3}$.

FIG. 5. The Helmholtz potential of a Reissner-Nordström hole as a function of the electrostatic potential (acting as an order parameter). At lower temperatures than the critical one there is a barrier (with a local maximum at $\Phi_{I}$ ) separating the metastable state $\Phi_{I I}$ from the ground state (with negative mass) $\Phi_{I I I}$. Thus, a first order transition is needed to pass from one to the other. As we rise the temperature, that barrier disappears allowing the transition be of second order. For higher temperatures only the state $I I I$ will be stable. 
Figure 1

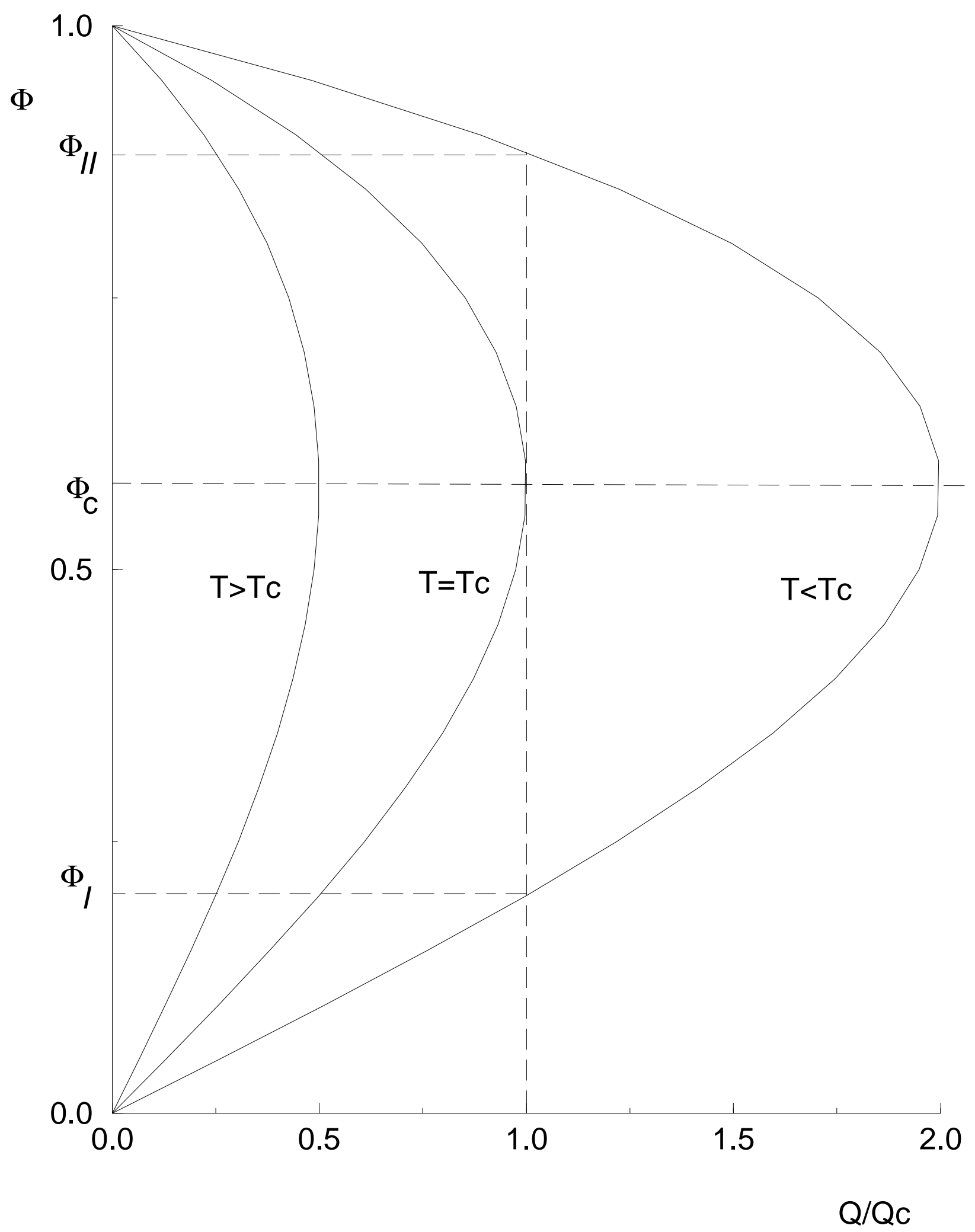


Figure 2

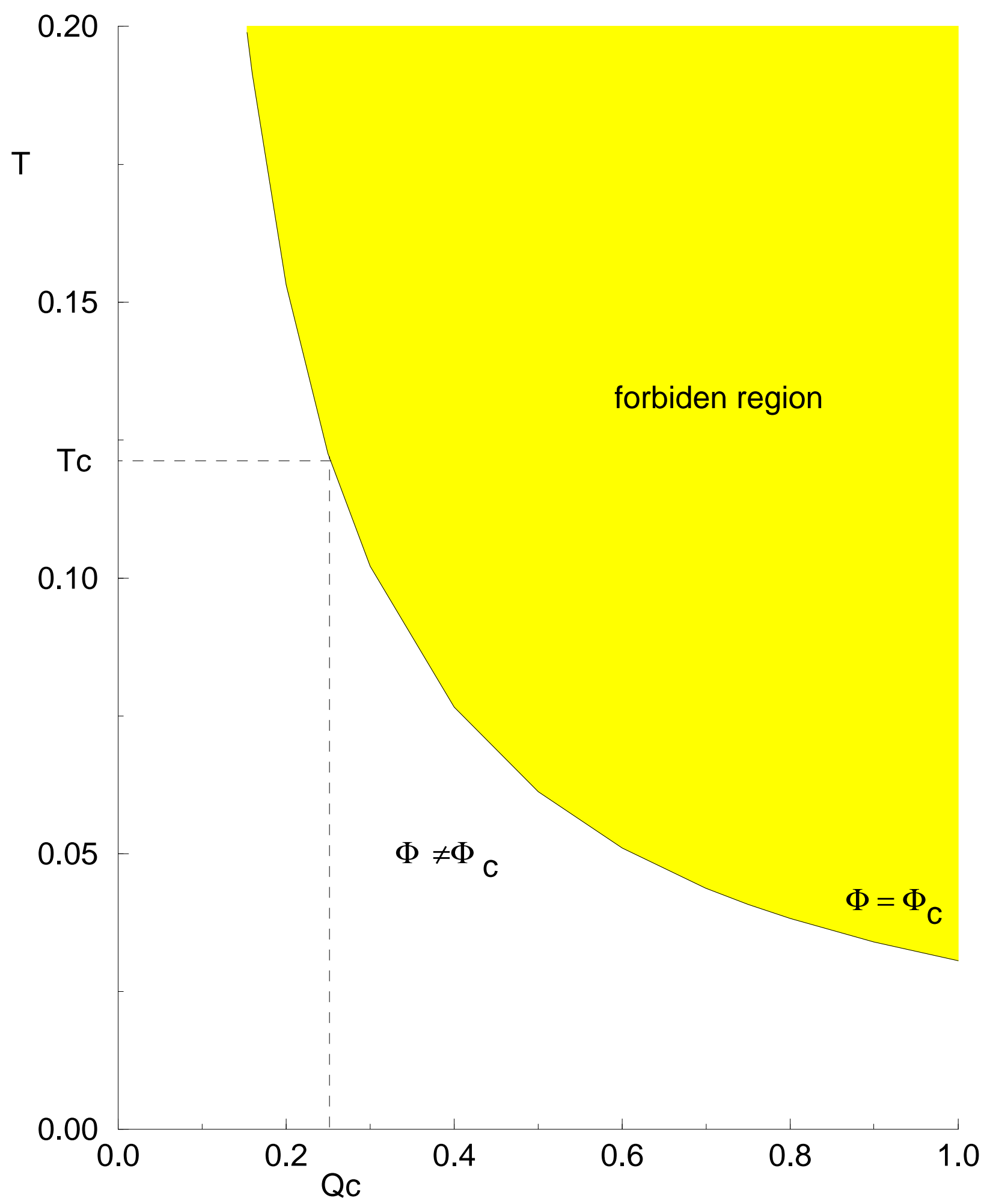


Figure 3

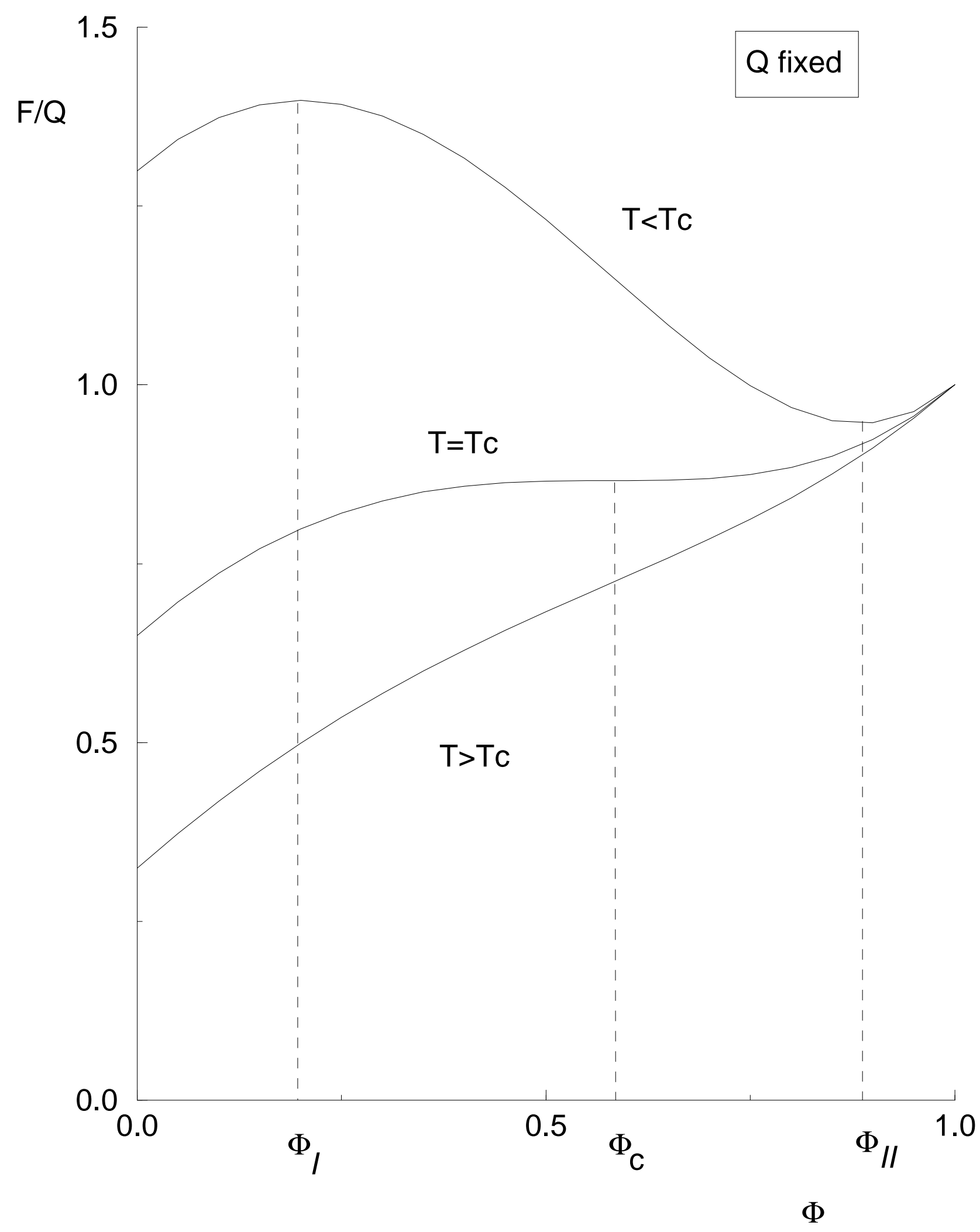


Figure 4

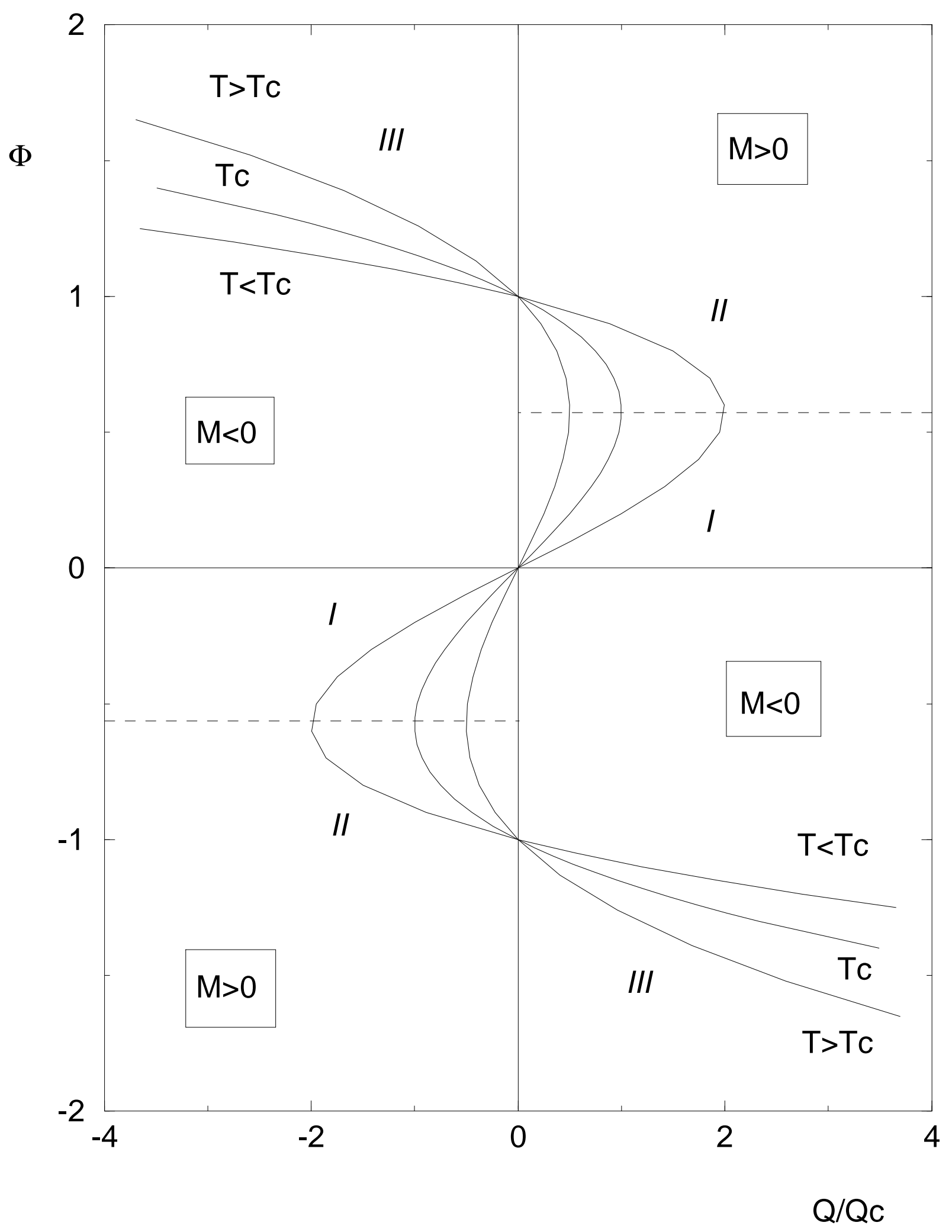


Figure 5

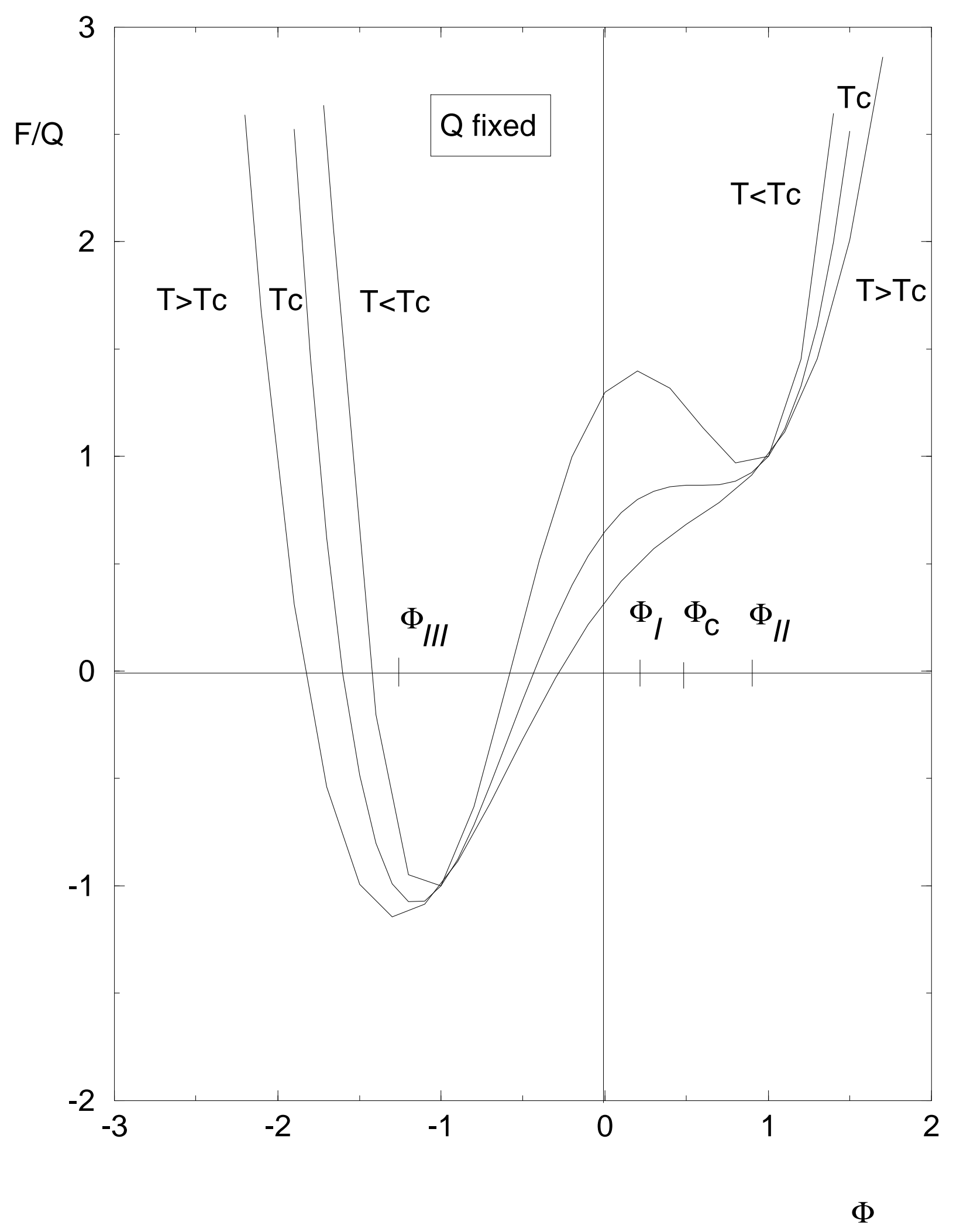

